\title{
The Effects of Holy Quran Recitation on Physiological Stress Response in Mechanically Ventilated Intensive Care Unit Patients: A Pilot Study
}

Nur Fariza Ramly ${ }^{1}$, Mohd Basri Mat Nor ${ }^{1}$, Azrina Md Ralib ${ }^{1}$, Nur Atiyah Ibrahim², Nor Adibah Mohd Hadzir², Nur Atiqah Zainol Bahar ${ }^{2}$

${ }^{1}$ Department of Anaesthesiology and Intensive Care, Kulliyyah of Medicine, International Islamic University Malaysia

${ }^{2}$ Kulliyyah of Medicine, International Islamic University

Presenter: Nur Fariza Ramly

Introduction: Mechanically ventilated intensive care unit (ICU) patients are exposed to stress due to the disease, intervention and surroundings. Pharmacological treatment is commonly used to reduce stress however it could give adverse effects. This study examined whether listening to Quranic recitation helps in reducing stress by observing the effects on vital signs, pain score, anxiety score and serum cortisol level. Methodology: Forty mechanically ventilated ICU patients of IIUM Medical Centre who fulfilled weaning criteria were randomised into Quran $(n=7)$ and control groups $(n=23)$. Patients in the Quran group received 30 minutes of uninterrupted bed rest while listening to Surah Al-Mulk via mp3 player followed by 30 minutes bed rest. For control group, 60 minutes of uninterrupted bed rest was allocated. APACHE score, SOFA score, pain score, anxiety score, serum procalcitonin and serum cortisol were taken before and after intervention. Heart rate $(\mathrm{HR})$, respiratory rate (RR), systolic (sBP) and diastolic (dBP) blood pressure were recorded at 5 minute intervals throughout the intervention period. Results: In the Quran group, vital signs showed decreasing trend from minute 0-30 but increased after minute 60 . Both pain and anxiety scores in Quran group showed decreasing trend throughout 60 minutes. In control, the vital signs, pain and anxiety score are unchanged. Serum cortisol in both groups showed increasing trend after the intervention. Conclusion: This preliminary finding demonstrated the potential effects of listening to Quranic recitation in reducing physiological stress response in mechanically ventilated ICU patients. The small sample size may explain decreasing trends but non-statistically significant results. 